\title{
Modern Mining Geostatistics-A Special Issue Dedicated to Our Friend Professor Danie Krige
}

\author{
Vaughan Chamberlain • Ian H. Douglas • \\ Edson Ribeiro • Malcolm L. Thurston • \\ Gavin Yeates • Roussos Dimitrakopoulos
}

Published online: 9 November 2013

(C) International Association for Mathematical Geosciences 2013

It is a privilege to introduce this Special Issue of Mathematical Geosciences on "Modern Mining Geostatistics", dedicated to our late colleague and friend Dr. Danie Krige, founding member of IAMG. Dr. Krige was a Professor of Mining Engineering at the University of Witwatersrand, and was known for his pioneering work in modelling and forecasting the spatial distribution of grades and metal content at producing mineral deposits. This field of research was eventually termed "Geostatistics". Professor Krige's efforts flourished over the decades to expand beyond mining, substantially influencing many fields of science and geo-engineering and becoming core elements in fields where spatial interpolation is required. These fields include environment, agriculture, soil science, water resources, climate modelling, biodiversity mapping, geographic information systems, petroleum and energy resources, and spatial statis-

V. Chamberlain

Anglo Gold Ashanti Ltd., Johannesburg, South Africa

I.H. Douglas

Newmont Mining Corporation, Denver, CO, USA

E. Ribeiro

Vale S.A., Belo Horizonte, Brazil

M.L. Thurston

De Beers, Johannesburg, South Africa

G. Yeates

BHP Billiton, Melbourne, Australia

R. Dimitrakopoulos ( $\bigotimes)$

COSMO_-Stochastic Mine Planning Laboratory, McGill University, Montreal, Canada

e-mail: roussos.dimitrakopoulos@mcgill.ca 
tics. A form of interpolation, "kriging", became a very familiar term in all the above and other scientific and engineering fields.

Mineral deposits are typically characterised by very sparse datasets and limited information. This serves to make the estimating and modelling of deposits both a critically important and technically complex task. It also provides a basis for mine planning and mineral reserves, production forecasting, and the sustainable development of the Earth's mineral resources. The latter development has become even more important today, due to the fast growth of new emerging economies and developing countries generating an ever-increasing demand for minerals; this coupled with increasing environmental concerns. Professor Krige's contributions offered the foundation upon which, over a half or so century later, modern technologies can offer a wealth of new innovative concepts. Powerful methods and approaches have provided a remarkable diversity of new frameworks and techniques for modelling orebodies, forecasting metal content and material types at any location in space, modelling the related uncertainty and integrating models of geological uncertainty to the strategic mine planning and sustainable development and utilisation of Earth's natural resources. In all, not exactly a commonplace contribution!

To celebrate Professor Krige's contributions, this Special Issue stresses today's Modern Mining Geostatistics and its advanced application. It serves as a demonstration of where the field stands due to the early work, continued contribution, commitment to the profession, and dedication to education and ethos of Professor Krige - the friend we will all remember as the textbook example of a real gentleman.

The first of the six papers on this Special Issue is by Jeff Boisvert, Mario Rossi, Kathy Ehrig, and Clayton Deutsch who deal with the modelling of the so-called geometallurgical properties of the orebody at the Olympic Dam Mine, South Australia. This is a challenging undertaking for several reasons, starting from the fact that mineral processing circuits are complex and depend on the interaction of a large number of properties of the ore feed, including the recoveries of $\mathrm{Cu}$ and $\mathrm{U}_{3} \mathrm{O}_{8}$, acid consumption, as well as empirical tests to measure the rock hardness and abrasiveness most of which do not average linearly to facilitate production forecasting. The authors propose a stochastic simulation framework that allows handling very large numbers of input variables to generate final models that are suitable for mine and plant optimisation.

Serge Séguret's paper follows, proposing a new indicator-based method for analysing and modelling multi-unit deposits and their product with the metal grade. The approach is demonstrated in an application at a porphyry copper deposit. Then, Peter Jones, Ian Douglas and Arja Jewbali present a fundamentally different approach to modelling geological units and grades based on multiple point statistics and stochastic simulation in an application at the structurally controlled Apensu Gold Deposit, Ghana. The paper shows that the simulated models generated are geologically realistic and display complex non-linear features in the deposit. A conceptual model of the deposit's geology is used as a training image to infer the high-order spatial relationships present. Multiple realisations of the Apensu deposit allow for an assessment of geological and volumetric uncertainty, which is further combined with grade simulations to generate a more complete picture of the true uncertainty of the deposit. 
Returning to more conventional Geostatistics, Jacques Rivoirard, Claude Demange, Xavier Freulon, Aurélie Lécureuil, and Nicolas Bellot visit the topic of heavytailed spatial datasets and propose a new model to deal with extreme values, based on splitting data values into three parts: the truncated grade, a weighted indicator above a top-cut grade and a residual. The proposed approach is then applied at both an actual gold deposit and on a simulated example to demonstrate that keeping the high values in the modelling process via an appropriate mathematical model is advantageous. The above is followed by the work of Raimon Tolosana-Delgado and Gerald van den Boogaart on the joint consistent mapping of high-dimensional geochemical surveys, where several tens of components, obtained from different horizons and with different analytical techniques are combined. To generate spatial elemental concentration maps that are of interest to mineral exploration, the authors suggest log-ratio multivariate analysis techniques that offer consistent solutions. Proposed procedures are illustrated using the horizon C Kola dataset, with 25 components and 605 samples covering most of the Kola peninsula (Finland, Norway, Russia).

The Special Issue concludes with well-known topic of forecasting recoverable ore reserves approached by a new method presented by Richard Peattie and Roussos Dimitrakopoulos, founded upon the stochastic simulation of future grade control drilling. The application of the proposed method at the Morila gold deposit, Mali, documents the new simulation-based approach for recoverable reserve forecasting that accounts for the potential effects of future grade control data. The approach accounts for both the potential grade control data that will become available as well as changes in data quantity and quality over time. As a result it allows for fully informed mining decisions to be made that incorporate the effects of information and selectivity while quantifying the potential impact of uncertainty on the mine operation and its final economic outcome.

We hope that you will enjoy the papers of this Special Issue!

The Guest Editors. 